\section{VULNERABILIDAD SÍSMICA HOSPITALARIA: EXPERIENCIAS DEL HOSPITAL REGIONAL DE ICA A CUATRO AÑOS DEL DESASTRE}

\section{HOSPITAL EARTHQUAKE VULNERABILITY: EXPERIENCES FROM THE HOSPITAL REGIONAL DE ICA WITHIN FOUR YEARS FROM THE DISASTER}

Reneé Pereyra-Elías ${ }^{1,2, a}$, Carmen Cecilia Elías-Barrera ${ }^{3,4, b}$, Nelson Morales-Soto ${ }^{5,6, c}$

Sr. Editor. Los hospitales son considerados como edificaciones esenciales y su falta de operatividad luego de un desastre supone un gran impacto social y político, ya que estos establecimientos constituyen uno de los pilares fundamentales que sustentan la respuesta ante tal situación ${ }^{(1)}$.

Como es de conocimiento público, luego del terremoto del año 2007 sucedido en el Perú, las instalaciones y líneas vitales del Hospital Regional de Ica colapsaron, ya que fue construido en la década de 1960, cuando aún no regía la norma de diseño sismorresistente. La atención continuó llevándose a cabo en el mismo lugar, en carpas y contenedores donados y en las más rescatables pero debilitadas estructuras que aún quedaban en pie. Dos años después, en agosto de 2009, los servicios de este establecimiento de salud fueron trasladados a un local del Instituto Peruano del Deporte, continuando en carpas y módulos prefabricados. Sin embargo, esta nueva locación tampoco cumplía con los requisitos necesarios para la labor asistencial.

Existieron muchas deficiencias en cuanto a las condiciones en las que se brindaba el servicio de salud: no se contó con el servicio de agua potable los primeros meses (el cual ahora es intermitente y deficiente), no existía un adecuado lugar para desechar la orina, otros fluidos y heces de los pacientes internados, la hospi-

\footnotetext{
Escuela de Medicina, Universidad Peruana de Ciencias Aplicadas. Lima, Perú.

2 Sociedad Científica de Estudiantes de Medicina de la Universidad Peruana de Ciencias Aplicadas (SOCIEMUPC). Lima, Perú.

3 Universidad Nacional San Luis Gonzaga de Ica. Ica, Perú.

4 Hospital Regional de Referencia de Ica. Ica, Perú.

5 Facultad de Medicina, Universidad Nacional Mayor de San Marcos. Lima, Perú.

6 Sociedad Peruana de Medicina de Emergencias y Desastres. Lima, Perú.

Estudiante de Medicina; ${ }^{b}$ Médico dermatólogo; ${ }^{c}$ Médico emergenciólogo.
}

Recibido: 18-03-11 Aprobado: 23-03-11 talización se realizó por un tiempo en carpas con piso de tierra, se carece hasta el día de hoy de un ambiente para el correcto mantenimiento de los cadáveres previo a su traslado, entre otras. Todo este contexto, aunque no ha sido medido, se convirtió en un potencial factor de riesgo para el desarrollo de infecciones y otras condiciones de diversa índole en contra de la calidad de atención al paciente.

En cuanto a la situación laboral, el personal de salud tuvo que realizar su trabajo en las circunstancias ya mencionadas, sobre pisos de tierra, ripio o, en el más afortunado de los escenarios, el cemento de lozas deportivas. Estos ambientes eran angustiosamente empeorados por el implacable clima soleado y seco de la región (siendo medidos más de $40^{\circ} \mathrm{C}$ en el interior de las carpas), no se contaba con servicios higiénicos exclusivos para el personal ni apropiadas áreas de descanso, etc.

Es importante resaltar la gran labor de todo el personal de salud que ha trabajado bajo estas condiciones durante estos casi cuatro años, atendiendo a más del $40 \%$ de la población del distrito de Ica, además de las otras regiones incluidas en su nivel de referencia (Ica departamento y departamentos vecinos).

Está próxima la reinstalación en el recientemente construido e implementado Hospital Regional de Ica, el cual cuenta con una moderna infraestructura. Del mismo modo, es probable que esté también próximo el olvido de la coyuntura vivida en estos años. Por ello, y tomando en cuenta la amenaza sísmica del litoral central del Perú (2), es de urgente necesidad que se enfatice e invierta más en programas de intervención del riesgo, especialmente mitigando los efectos destructivos de un potencial desastre y haciendo los preparativos con los que se afrontarán los daños inexorables que sucedieren (3). Además, se debe actualizar y extender los estudios de vulnerabilidad hospitalaria, evaluando tanto la estructura física como el componente organizativo, en busca del logro de más "hospitales seguros", que mantengan su máxima capacidad de atención en su misma infraestructura luego de un desastre ${ }^{(1,4)}$. Esto es de vital importancia para que una experiencia similar no se repita.

\section{Fuentes de Financiamiento.}

Autofinanciado.

\section{Conflictos de Interés.}

Los autores declaran no presentar conflictos de interés.

\section{REFERENCIAS}

1. Bambaren C, Alatrista MS. Hospitales seguros ante desastres. Rev Med Hered. 2007;18(3):149-54. 
2. Morales-Soto N, Zavala C. Terremoto en el litoral central del Perú: ¿Podría ser Lima el escenario de un futuro desastre? Rev Peru Med Exp Salud Publica. 2008; 25(2): 21724.

3. Morales-Soto N, Gálvez-Rivero W, Chang-Ausejo C, Alfaro-Basso D, García-Villafuerte A, Ramírez-Maguiña M, et al. Emergencias y desastres: desafíos y oportunidades (de la casualidad a la causalidad). Rev Peru Med Exp Salud Publica. 2008;25(2):237-42.

4. Morales-Soto N, Sato-Onuma J. Vulnerabilidad del componente organizativo y funcional de grandes hospitales. Rev Peru Med Exp Salud Publica. 2008;25(2):225-9.

Correspondencia: Reneé Francisco Pereyra Elías.

Dirección: Jr. Alonso de Molina \#1387 - Dpto 302. Urbanización

Polo Hunt - Monterrico, Lima 33, Perú.

Teléfono: (511) 345-2220 / (511) 981-213381.

Correo electrónico: renee.pereyra.elias@gmail.com

\section{LA DESNUTRICIÓN EN LA NIÑEZ EN EL PERÚ: FACTORES CONDICIONANTES Y TENDENCIAS}

\section{CHILDHOOD MALNUTRITION IN PERU: CONDITIONANT FACTORS AND TRENDS}

\author{
Wilfredo Gutiérrez
}

Sr. Editor. El término malnutrición proteico-energética, se ha utilizado para describir una serie de trastornos caracterizados, principalmente, por el retraso del crecimiento de niñas y niños. Sin embargo, este retraso en el crecimiento es solo una manifestación de una serie de afecciones al desarrollo físico y mental. Este tipo de desnutrición tiene mayor gravedad en menores de tres años, debido a sus elevadas necesidades energéticas, proteicas y a su particular vulnerabilidad a las infecciones.

Hoy en día existen suficientes evidencias de que la desnutrición, aun en sus formas moderadas, puede incrementar la probabilidad de morir por un número variado de enfermedades y puede estar asociada con cerca de la mitad de toda la mortalidad infantil (1). Igualmente, se tiene evidencias suficientes de que la desnutrición fetal

Instituto CUANTO. Lima, Perú

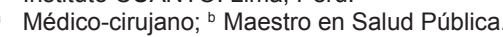

Recibido: 19-03-11 Aprobado: 23-03-11 y en las etapas tempranas de la vida de la niña o del niño, conlleva a daños permanentes en el crecimiento del cerebro, que son irrecuperables aun después de una realimentación prolongada ${ }^{(2)}$. Las consecuencias de desnutrición temprana en niñas y niños persisten en los años de la escuela y en la vida adulta, disminuyendo la productividad y la calidad de vida.

El estado nutricional es el resultado de una amplia gama de condiciones sociales y económicas y constituye un indicador muy sensible del nivel general de desarrollo. El marco conceptual de las causas de la malnutrición difundido por UNICEF plantea tres grandes grupos: a) Causas inmediatas, las relacionadas con la ingesta inadecuada de alimentos y micronutrientes y las enfermedades de tipos infeccioso; b) Causas subyacentes, relacionadas con la inseguridad alimentaria en el hogar, que tiene que ver con la poca disponibilidad de alimentos, la falta de acceso a ellos y su uso inadecuado, que a su vez, tiene ver con patrones de inadecuada información y c) Causas básicas, relacionadas con las estructuras políticas, económicas e ideológicas en la sociedad que regulan el acceso a los recursos, la educación y las oportunidades y que finalmente determinan la pobreza de las familias, que es la raíz del problema.

En el Perú, según el estudio de análisis del INEI y Prisma de las bases de datos de las ENDES 92 y $96^{\text {(3), las }}$ principales causas con fuerte asociación a la desnutrición crónica incluyen: la falta de control prenatal en la madre; el tener cuatro o más hijos vivos, que representa cuatro veces más la probabilidad de tener una niña o niño desnutrido; el bajo peso al nacer, que aumenta en dos veces el riesgo de desnutrición; el nivel de instrucción de la madre - el analfabetismo o primaria incompleta aumentan cuatro y dos veces más el riesgo de desnutrición respectivamente-; la carencia de servicios higiénicos o la tenencia de letrinas, que incrementa en tres veces más la probabilidad de desnutrición de la niña o niño; el hecho que la niña o niño sea cuidado por una mujer mayor de 40 años, la cual duplica el riesgo de desnutrición; el tener viviendas con piso de tierra que incrementa el riesgo en un $65 \%$, debido, probablemente, a que el niño está más propenso a adquirir infecciones; y la presencia de enfermedad diarreica aguda, la cual se asocia con un incremento del $30 \%$ en el riesgo de desnutrición.

El grupo Apoyo, en un estudio similar al de Prisma ${ }^{(4)}$, muestra que la edad de 6 a 18 meses es la más frecuente para que una niña o niño se desnutra; la ENDES 2000 agrega que el orden de nacimiento cumple una función importante como causa de malnutrición crónica, siendo casi el triple la prevalencia de desnutrición en aquellos que ocupan el sexto lugar con relación a los que ocupan el primero. 